(Aus dem physiologischen Institut der Cniversität Rostock.)

\title{
Über \\ die Wirkung erhöhter Temperatur auf die Reflexerregbarkeit des Froschrückenmarks.
}

Von

\section{H. Geinitz und H. Winterstein.}

Mitgeteilt von $\mathrm{H}$. Winterstein.

Die Wirkung erhöhter Temperatur auf die Reflexerregbarkeit ist bereits mehrfach Gegenstand der Beobachtung gewesen ${ }^{1}$ ). Besonders die merkwürdige Erscheinung der Wärmelähmung hat ein eingehenderes Studium erfahren ${ }^{2}$ ). Eine genauere Untersuchung des Verhaltens der Reflexerregbarkeit vor ihrem Erlöschen aber wurde bis jetzt nicht vorgenommen. Zur Feststellung desselben bedienten wir uns der elektrischen Reizung als der am leichtesten abstufbaren, und zwar der Reizung des peripheren Nervenstammes durch tetanisierende Induktionsströme, da beim normalen Frosch Einzelschläge nicht oder doch nur sehr unregelmässig wirksam sind und von der Haut her durch elektrische Reize nur schwer eine Reaktion zu erzielen ist. Um eine stets gleiche Zahl von Reizen einwirken zu lassen und so genau vergleichbare Werte zu erhalten, bedienten wir uns eines Verfahrens, welches schon $\mathrm{Mulert}{ }^{3}$ ) im hiesigen Institute angewendet hatte, und welches darin besteht, dass in den sekundären Stromkreis eines Schlitteninduktoriums eine rotierende Trommel eingeschaltet wird, welche mittels eines Schleif-

1) Vgl. O. Langendorff, Physiologie des Rücken- und Kopfmarks, in Nagel's Handbuch der Physiologie Bd. 4 Teil 1 S. $248 \mathrm{ff}$.

2) H. Winterstein, Über die Wirkung der Wärme usw. Zeitschr. f. allgem. Physiol. Bd. 1 S. 129. - Wärmelähmung und Narkose. Ebenda Bd. 5 S. 323.

3) G. Mulert, Über elektrische Reizung des Halssympathicus. Pflüger's Arch. Bd. 55 S. 550.1894. 
kontaktes die Schliessung dieses Stromkreises für eine stets gleiche Zeit - in unseren Versuchen etwa $1 / 4$ Sekunden - bewirkt. Der Strom wurde von einem Akkumulator geliefert.

Als Reflexpräparat diente anfänglich das nach Baglioni ${ }^{1}$ ) oder auch das völlig isolierte ${ }^{2}$ ) Rückenmark, das in einer Atmosphäre von Sauerstoff oder in einer mit Sauerstoff gesättigten Kochsalzlösung gehalten wurde; die späteren Versuche wurden jedoch, um möglichst normale Bedingungen zu erhalten und vor allem den Einfluss einer etwaigen Erstickung bei höherer Temperatur durch ungenügende Sauerstoffzufuhr auszuschalten, am ganzen Frosch vorgenommen, dem meist tags zuvor das Rückenmark durchschnitten worden war. Nach Unterbindung der Femoralarterien wurden die beiden Ischiadici freigelegt, der eine mittels Platinelektroden gereizt und als Index die Zehen oder der mit einem leichten Hebel verbundene Gastrocnemius des anderen Beines verwendet. Das in den ersten Versuchen geübte Biedermann'sche Verfahren ${ }^{3}$, , bei welchem der Reflex vom Ischiadicus auf den Triceps femoris der gleichen Seite als Index dient, wurde verlassen, als wir beobachteten, dass hierbei eine Täuschung durch sekundäre Erregung nicht ausgeschlossen ist ${ }^{4}$ ).

Als Mass der Erregbarkeit diente die Reizschwelle, die in einem Zeitintervall von meist 10 Minuten aufgesucht wurde. Um das Präparat nicht durch wiederholte Reizung beim Feststellen der Reizschwelle zu ermüden, wurde diese nicht auf den Zentimeter genau bestimmt, sondern die Rolle des Induktoriums jedesmal gleich um $3 \mathrm{~cm}$ weitergerückt. Auf diese Weise genügten meist wenige Reizungen zur Bestimmung der Grenzen, zwischen welche die Reizschwelle fiel. War z. B. beim Rollenabstande 50 noch eine Reaktion

1) S. Baglioni, La fisiologia del midollo spinale isolato. Zeitschr. f. allgem. Physiologie Bd. 4 S. 384.

2) H. Winterstein, Zur Frage der Sauerstoffspeicherung. Zentralbl. f. Physiologie Bd. 20 S. 41. 1906.

3) W. Biedermann, Beiträge zur Kenntnis der Reflexfunktion des Rückenmarks. Pflüger's Arch. Bd. 80 S. 408. 1900.

4) Wir sahen in zwei Fällen nach völliger Abtrennung des. Beines bei Reizung des Ischiadicus durch einen schwachen Induktionsschlag eine Reaktion des Triceps erfolgen. Da diese Erscheinung nicht auf Stromschleifen zurückzuführen war, so kann es sich wohl nur um eine wahre sekundäre Erregung des den Triceps versorgenden Nervenastes durch die negative Schwankung des Ischiadicus gehandelt haben, wie dies von Hering (Sitzungsber. d. Wiener Akad. d. Wissensch. Bd. 85. 1883) beschrieben wurde. 
Über die Wirkung erhöhter Temperatur auf die Reflexerregbarkeit etc. 275 auslösbar, bei 53 hingegen nicht mehr, so wurde $50-53$ als Reizschwelle angeschrieben. - Die Erwärmung erfolgte in einem Wasserbad, in welches das das Präparat und das Thermometer enthaltende Glasgefäss getaucht war.

Das Resultat unserer Versuche war ein höchst unerwartetes. Wohl alle bisherigen Beobachter, auch ich selbst, glaubten in der Wärme eine sehr bedentende Steigerung der Reflexerregbarkeit beobachten zu können. Die Bestimmung der Reizschwelle für elektrische Reizung ergab hingegen, dass entweder gar keine oder nur eine sehr unbeträchtliche Steigerung der Erregbarkeit (Vergrösserung des Rollenabstandes um wenige Zentimeter) auftritt. Einige Versuchsprotokolle mögen dieses Verhalten erläutern:

Versuch 40. (6. Juli 1906.)

Rana esculenta. Isoliertes Rückenmark in Sauerstoffatmosphäre. Reizung des Ischiadicus der einen, Beobachtung des mit einem Strohhebel verbundenen Gastrocnemius der anderen Seite.

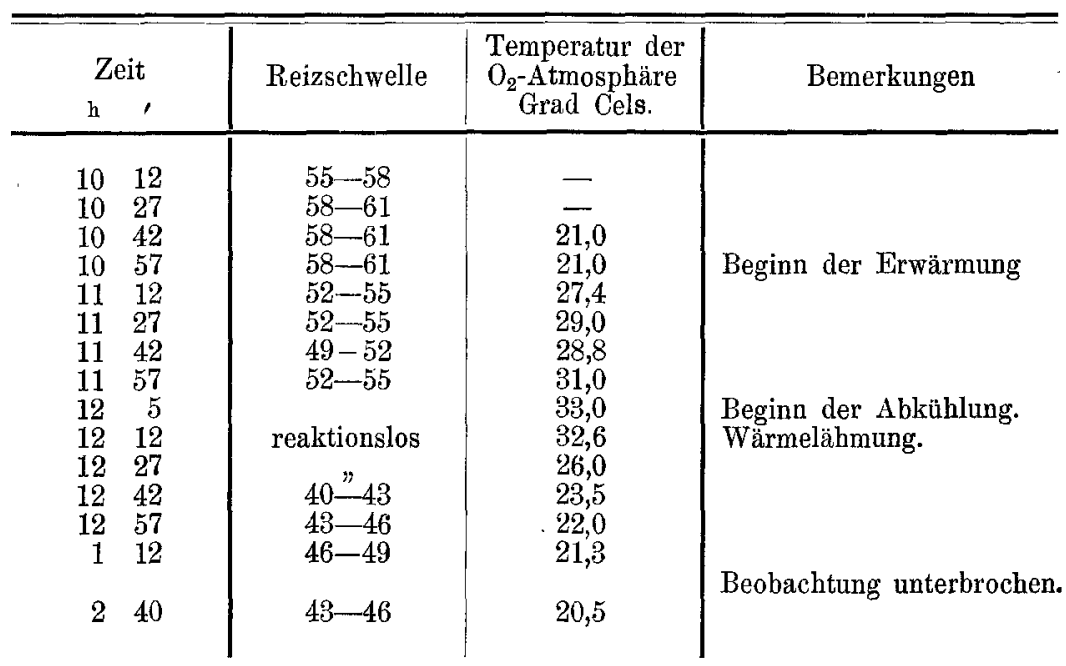

Bei diesem Versuch wurde zwischen zwei Bestimmungen der Reizschwelle jedesmal eine Reflexzuckung des Gastrocnemius bei einem Rollenabstand von $40 \mathrm{~cm}$ verzeichnet. Die Zuckungen zeigten die gleiche Höhe bis zum Verschwinden der Erregbarkeit in der Wärmelähmung.

Versuch 45. (27. Juli 1906.)

Rana esculenta. Rüekenmark tags zuvor durchschnitten. Reizung des Ischiadicus der einen, Beobachtung des mit einem Strohhebel verbundenen 
Gastrocnemius der anderen Seite. Temperatur gemessen in dem den Frosch enthaltenden Gefäss.

\begin{tabular}{|c|c|c|c|c|}
\hline $\begin{array}{l}\mathrm{Zei} \\
\mathbf{h}\end{array}$ & , & Reizschwelle & $\begin{array}{l}\text { Temperatur } \\
\text { Grad Cels. }\end{array}$ & Bemerkungen \\
\hline $\begin{array}{l}9 \\
10 \\
10 \\
10 \\
10 \\
10 \\
10 \\
11 \\
11 \\
11 \\
11\end{array}$ & $\begin{array}{l}50 \\
00 \\
10 \\
20 \\
30 \\
40 \\
50 \\
00 \\
10 \\
20 \\
30\end{array}$ & $\begin{array}{c}57-60 \\
60-63 \\
60-63 \\
60-63 \\
60-63 \\
63-66 \\
60-63 \\
57-60 \\
60-63 \\
60-63 \\
\text { reaktionslos }\end{array}$ & $\begin{array}{l}- \\
\overline{-} \\
20,0 \\
20,6 \\
24,0 \\
24,6 \\
25,8 \\
29,0 \\
32,2 \\
35,0\end{array}$ & $\begin{array}{l}\text { Beginn der Erwärmung } \\
\\
\text { Wärmelähmung. } \\
\text { Bei Abkühlung tritt keine } \\
\text { Erholung mehr ein. }\end{array}$ \\
\hline
\end{tabular}

Versuch 57. (11. August 1906.)

Rana esculenta. Rückenmark tags zuvor durchschnitten. Versuchsanordnung wie im vorangehenden Experiment.

\begin{tabular}{rr|c|c|l}
\hline \multicolumn{2}{c|}{$\begin{array}{c}\text { Zeit } \\
\text { h }\end{array}$} & Reizschwelle & $\begin{array}{c}\text { Temperatur } \\
\text { Grad Cels. }\end{array}$ & Bemerkungen \\
\hline 11 & 45 & $60-63$ & - & \\
11 & 55 & $57-60$ & - & \\
12 & 5 & $57-60$ & - & \\
12 & 15 & $57-60$ & 19,5 & Beginn der Erwärmung. \\
12 & 25 & $54-57$ & 22,0 & \\
12 & 35 & $54-57$ & 25,0 & \\
12 & 45 & $57-60$ & 29,0 & \\
12 & 55 & $60-63$ & 34,0 & Beginn der Abküblung. \\
1 & 5 & $47-50$ & 37,0 & Wärmelähmung. \\
1 & 15 & reaktionslos & 30,0 & \\
1 & 25 & $n$ & 24,0 & Beobachtung unterbrochen. \\
3 & 55 & $47-50$ & 21,5 & \\
4 & 5 & $47-50$ & - & \\
4 & 15 & $47-50$ & - &
\end{tabular}

Das überraschende Ergebnis dieser Versuche führt zu der Frage, wodurch der Eindruck einer so bedeutenden Steigerung der Erregbarkeit bei mechanischer Reizung des erwärmten Frosches entsteht? Eine völlig befriedigende Antwort auf diese Frage vermag ich nicht zu geben. Man könnte daran denken, dass tatsächlich eine Steigerung der Erregbarkeit der sensiblen. Nervenenden der Haut eintritt. Ich glaube jedoch, dass die Erregbarkeitssteigerung der Hauptsache nach nur vorgetäuscht wird durch die grosse Schnelligkeit und ausser- 
ordentliche Intensität, mit welcher eine jede Aktion der erwärmten Muskeln erfolgt. Dadurch treten Reaktionen zutage, die sonst wegen ihrer Langsamkeit und Geringfügigkeit nicht in die Augen fallen, und alle Bewegungen erscheinen in vergrössertem Masse.

Aber auch die Frage nach der Natur des der Wärmelähmung vorangehenden Erregungsstadiums drängt sich aufs neue auf, da es paradox erscheinen muss, dass ein Erregungsstadium ohne erhebliche Steigerung der Erregbarkeit auftreten kann. Von der Auffassung ausgehend, dass die Wärmelähmung eine Erstickung darstelle, habe ich das ihr vorangehende Erregungsstadium als eine Art Wärmedyspnoe gedeutet ${ }^{1}$ ), die nicht so sehr der absoluten Höhe der Temperatur als dem durch sie erzeugten Zustande der Asphyxie ihre Entstehung verdanke. $\mathrm{Carlson}^{2}$ ), der meine Deutung der Wärmelähmung wenigstens für das Herz für unzutreffend hält, gibt an, dase der enthirnte Froseh bei langsamer Erwärmung kein Erregungsstadium zeige, und dass dieses daher bloss die letzte Willensanstrengung darstelle, aus der unangenehmen Atmosphäre zu entkommen; nur bei plötzlicher Erwärmung würde auch der „Spinalfrosch" Erregungserscheinungen darbieten. Diese merkwürdige Angabe schien mir eine Nachprüfung zu erfordern, und ich beschloss daher, systematisch zu untersuchen, ob das Erregungsstadium der Wärme dem ganzen Zentralnervensystem eigentümlich ist oder an die Existenz eines bestimmten Teiles desselben gebunden ist. Die Versuche wurden an Temporarien und Esculenten angestellt, die meist erst am Tage nach der in Narkose vorgenommenen Operation, nachdem sie sich von dem Eingriff völlig erholt hatten, erwärmt wurden. Sie gaben übereinstimmend das folgende Resultat:

Wenn man bei einem Frosch das ganze Gehirn bis hart an die Medulla oblongata entfernt, so wird das Erregungsstadium der Wärme hierdurch in keiner Weise beeinflusst, sofern die Medulla oblongata selbst nicht so verletzt ist, dass die Atmungstätigkeit aufgehört hat. Ein solcher Frosch unterscheidet sich in seinem Verhalten bei der Erwärmung in nichts von einem normalen. Ist hingegen die Medulla oblongata selbst durchschnitten oder verletzt

1) H. Winterstein, Wärmelähmung und Narkose, a. a. 0. S. 348.

2) A. J. Carlson, Temperature and heart activity with special reference to the heat standstill of the heart. Aneric. Journ. of Physiol. vol. 15 p. 207. 1906. 
worden und die Atmung erloschen, so tritt die Wärmelähmung ohne eine Spur spontaner Erregung ein, gleichviel ob die Erwärmung rasch oder langsam erfolgt. Durchschneidet man das Rückenmark unterhalb der Medulla oblongata, so zeigt der mit dieser verbundene Teil des Körpers heftige Erregungserscheinungen, während die hintere Körperbälfte bis zum Eintritt der Wärmelähmung vollkommen regungslos verharrt.

Dieses sehr bemerkenswerte Versuchsergebnis lehrt also, dass die Ansicht Carlson's, das Erregungsstadium verdanke seine Entstehung Grosshirnimpulsen, irrig ist. Nicht das Gehirn, sondern die Medulla oblongata und zwar, wie wir mit grösster Wahrscheinlichkeit annehmen dürfen, das Atemzentrum ist der Ausgangspunkt desselben. Durch die Beobachtung, dass das Atemzentrum, das ja in der Tat in erster Linie seine Beeinflussung durch die Wärme durch beschleunigte Tätigkeit dokumentiert, das Erregungsstadium der Wärmewirkung auslöst, erfährt meine Auffassung, dass es sich hierbei um eine Form von Dyspnoe handle, eine unerwartete und gewiss sehr beweiskräftige Stütze.

Der scheinbare Widerspruch eines Erregungsstadiums ohne gesteigerte Erregbarkeit würde sich demnach dahin aufklären, dass die letztere sich ausschliesslich oder wenigstens der Hauptsache nach auf das verlängerte Mark beschränkt. Es bleibt noch der tetanische Charakter ${ }^{1}$ ) zu erklären, den das Erregungsstadium an seinem Ende anzunehmen pflegt, und der sonst gleichfalls ein Kennzeichen ausserordentlich gesteigerter Reflexerregbarkeit darstellt. Ich glaube nun, dass es sich hierbei nicht, wie bei der Strychninvergiftung um eine gleichzeitige Innervation der Strecker und Beuger handelt, sondern hauptsächlich darum, dass die Zentren der Beugemechanismen früher der Wärmelähmung verfallen als jene der Streckbewegungen, wodurch diese das Übergewicht erhalten und der Eindruck tetanischer Krämpfe erzeugt wird. In der Tat kann man bei genauer Beobachtung nicht selten feststellen, dass den ausgiebigen Streckbewegungen rudimentäre, wegen der bereits vorgeschrittenen Schädigung der Zentren nur sehr unvollkommene Beugeversuche nachfolgen. Diese Auffassung erklärt, warum der tetanische Charakter des Erregungsstadiums immer erst zum Schluss, meist kurz vor der völligen Wärmelähmung, wahrnehmbar wird, denn er würde eben

1) Vgl. H. Winterstein, Über die Wirkung der Wärme usw., a. a. O. 
bereits den Beginn der letzteren darstellen; auch meine sonst nur schwer zu erklärende Beobachtung ${ }^{1}$ ), dass diese charakteristische Form des Erregungsstadiums nur bei allmählicher Erwärmung deutlich zum Vorschein kommt, bei raschem Ansteigen der Temperatur hingegen oft fehlt, wird hierdurch verständlich: der tetanische Charakter des Erregungsstadiums wird nur dann hervortreten können, wenn zwischen der Lähmung der zentralen Beuge- und der Streckmechanismen eine längere Zeit vergeht; werden beide fast gleichzeitig gelähmt, wie dies bei raschem Erhitzen der Fall ist, so kann auch diese Eigentümlichkeit des Erregungsstadiums nicht zur Entfaltung kommen.

Im Anschluss an die Besprechung dieser Versuche sei noch erwähnt, dass wir auch eine Versuchsreihe mit Abkühlung des isolierten Rückenmarks bis auf und unter $0^{\circ}$ angestellt haben, ohne hierbei eine Steigerung der Reflexerregbarkeit beobachten zu können. Doch ist die Zahl der Versuche nicht ausreichend, um weitere Schlussfolgerungen aus ibnen zu ziehen.

\section{Zusammenfassung.}

1. Die Erhöhung der Temperatur des Froschrückenmarks bewirkt keine oder nur eine sehr geringfügige Steigerung der Reflexerregbarkeit.

2. Das der Wärmelähmung vorangehende Erregungsstadium wird ausgelöst von der Medulla oblongata, sehr wahrscheinlich vom Atemzentrum; nur die mit diesem verbundenen Teile des Zentralnervensystems zeigen Erregungserscheinungen.

Zum Schlusse sagen wir Herrn Professor Langendorff für seine liebenswürdigen Ratschläge unsern verbindlichsten Dank.

1) H. Winterstein, Über die Wirkung der Wärme usw., a, a. 0 . 